\title{
Changing Pattern of Congenital Heart Disease Care During COVID-19 Pandemic
}

\author{
Sakshi Sachdeva ${ }^{1}$ - Anita Saxena ${ }^{1,2} \cdot$ Samir Shakya ${ }^{1}$ - Sivasubramanian Ramakrishnan ${ }^{1} \cdot$ Saurabh K. Gupta ${ }^{1}$. \\ Shyam S. Kothari ${ }^{1}$
}

Received: 5 November 2020 / Accepted: 9 February 2021 / Published online: 23 March 2021

(C) Dr. K C Chaudhuri Foundation 2021, corrected publication 2021

\begin{abstract}
Objective To study the impact of coronavirus disease 2019 (COVID-19) pandemic on the utilization of pediatric cardiac care services and to determine the role of teleconsultation services in delivering healthcare in this subset of population.

Methods It was a retrospective, observational study. All children who attended pediatric cardiology outpatient/teleconsultation services or were admitted to pediatric cardiology ward between April 1, 2019 to July 31, 2019 and April 1, 2020 to July 31, 2020, were recruited in the study. Data for patients who underwent surgery or catheter intervention for congenital heart disease were also recorded and analyzed. Comparisons were drawn between the statistics during the two time-periods.

Results Physical outpatient services were discontinued and were replaced by teleconsultations from April 2020. Inpatient admissions during COVID-19 pandemic $(\mathrm{n}=66)$ decreased by two-thirds as compared to the admissions during similar period in $2019(\mathrm{n}=189)$. Similarly, the percentage decrease during these 4 mo of pandemic were $84 \%$ for catheter interventions, $88 \%$ for total congenital heart disease (CHD) surgeries, and $40 \%$ for emergency CHD surgeries. The number of patients availing successful teleconsultation was 1079 , which was only $15 \%$ of the total number of patients attending physical outpatient services ( $n=7176$ ) during the corresponding period in the year 2019. During the pandemic, systematic teleconsultation and local evaluation and investigations aided in better management of patients with CHD.

Conclusions The utilization of cardiovascular services for CHD has reduced significantly during COVID-19 pandemic, for both out- and inpatient care. Teleconsultation services have streamlined the follow-up care to some extent and have helped in noncontact triaging of these patients for further care.
\end{abstract}

Keywords COVID-19 $\cdot$ Congenital heart disease $\cdot$ Pediatric cardiology $\cdot$ Teleconsultation

\section{Introduction}

Coronavirus disease 2019 (COVID-19) pandemic diverted entire healthcare infrastructure towards tackling the pandemic in its initial phase. The already crunched congenital heart disease (CHD) care infrastructure in resource-limited settings faced a major setback [1]. Children with critical heart disease needing urgent care were not able to reach the facility due to transportation issues and

Anita Saxena

anitasaxena@hotmail.com

1 Department of Cardiology, All India Institute of Medical Sciences, New Delhi, India

2 Department of Cardiology, 7th Floor, Cardio Thoracic Science Centre, All India Institute of Medical Sciences, New Delhi 110029 , India lockdowns. Many CHDs need early intervention and may turn fatal or inoperable, if not dealt with in time. Considering this, modified timing of interventions as per the physiological and hemodynamic requirements were proposed $[2,3]$.

For providing adequate distancing between patients, proportionate reductions in number of beds for patient care were made. Elective diagnostic tests including echocardiography and cardiovascular computed tomography (CT), which are important imaging modalities in the evaluation of children with CHD, were deferred for many patients [4]. Barring emergency procedures, most of the centers suspended all the elective diagnostic catheterization, interventions, and cardiac surgeries. Also, due to public health measures of social distancing in place, children and adults with CHD were not able to carry out regular physical activities, which could affect their cardiovascular health [5]. Few studies have reported $40 \%$ reductions in admissions for acute myocardial 
infarctions and acute heart failure [6, 7]. But the magnitude of reduction in pediatric cardiac care is not known.

From a tertiary care center in North India, the authors share their experience regarding the care of children and adults with CHDs during COVID-19 pandemic over the 4 mo period of national lockdown. First COVID-19 case was reported from India in January 2020, and nation-wide lockdown came into effect from 23 March 2020. Data are presented for the period of 4 mo from April 1, 2020 to July 31, 2020 and are compared with the corresponding period in the year 2019 .

\section{Material and Methods}

This is a retrospective, observational study. The authors reviewed their outpatient and inpatient records during the study period. Ethical approval was obtained from the institutional ethics committee.

Teleconsultation services in pediatric cardiology were offered for $48 \mathrm{~d}$ during the period between April 1 and July 31, 2020. During the initial $2 \mathrm{mo}$, patients already registered in the hospital and seen in the outpatient department before the lockdown period were sent text messages on their registered phone numbers and they were advised to call for teleconsultation during specified time slots on specific days (which corresponded to earlier outpatient timings and days). In the second half of this time period, patients were encouraged to take appointments through the centers' website or through interactive voice response system, in addition to the earlier system of messaging. These patients were then called on their registered phone number on the appointed date and time. The senior resident doctors (with at least 2 y of experience in pediatric cardiology) communicated with all the parents/ patients and asked about the welfare of the patient. After knowing the hospital registration number (UHID), the echocardiogram report was retrieved from the hospital database. Based on the information obtained, advice was given. In case of any doubt, opinion from the consultant of pediatric cardiology was obtained and the plan thus made was communicated to the patient. Investigations as required were performed locally and the treatment plan was modified accordingly. If considered necessary, the patients were asked to visit hospital so that they could be examined physically. The data so obtained was compared with similar period in the year 2019 .

All admissions were made through the pediatric emergency department. Patients reporting to emergency department were assessed for need of admission. Those requiring admission were tested for COVID-19. If found COVID positive, they were kept in a COVID-designated area. Those tested negative were admitted to pediatric cardiology ward or pediatric cardiology intensive care depending on their clinical status.
Patients were also admitted through teleconsultation route, if they tested negative for COVID. In case an attendant was required to stay with the child, COVID-19 test was done for the attendant also. Patients with cyanotic CHD with deep cyanosis/spells or heart failure, critical/severe obstructive lesions, like aortic stenosis/pulmonary stenosis/prosthetic heart valve stenosis/coarctation of aorta, and infective endocarditis were labelled as emergency cases.

All indoor patients underwent echocardiography after admission in the ward. The healthcare staff caring for the indoor patients were advised to take adequate precautions in the form of wearing N95 masks, water-impermeable gown, shoe cover and head cap. Face-shields and goggles were used in addition when performing any procedure on the child including cardiac catheterization. The patients with confirmed diagnosis by echocardiogram, were categorized into subgroups, depending upon the hemodynamic subset of the lesion. The data of these 4 mo were compared with similar period in 2019.

Data regarding number of catheterization, interventions, and cardiac surgery performed during the 4 mo period of COVID pandemic were collected from the hospital records. A comparison was made for the corresponding duration of 4 mo in the year 2019.

Statistical analysis: Analysis was done using Microsoft Excel 2013 (Microsoft Corp). The percentage decrease in number of total CHD surgeries, emergency CHD surgeries, and catheter interventions during the current year was expressed using the formula [(number in 2019 - number in 2020)/number in $2019] \times 100$.

\section{Results}

There was a significant reduction in the number of patients who received outpatient or inpatient care as well as those who underwent surgical or transcatheter interventions (Table 1).

Table 1 Comparison of number of patients utilizing inpatient and outpatient pediatric cardiac care services between April 1 and July 31, in 2020 and 2019

\begin{tabular}{llll}
\hline & $2020(\mathrm{n})$ & $2019(\mathrm{n})$ & Percent reduction \\
\hline OPD/Teleconsultation & 1079 & 7176 & $84.9 \%$ \\
Indoor admissions & 66 & 189 & $65 \%$ \\
Total CHD surgeries & 50 & 409 & $87.8 \%$ \\
Emergency CHD surgeries & 35 & 58 & $39.6 \%$ \\
Transcatheter intervention & 14 & 87 & $83.9 \%$ \\
Diagnostic catheterization & 2 & 131 & $98.4 \%$ \\
\hline
\end{tabular}

CHD Congenital heart disease; $O P D$ Outpatient department 
During this 4-mo period, 1190 patients/parents were contacted. Of these, 1079 (90.6\%) calls were successful. The reasons for failure to contact the patient included incorrect phone number $(n=102)$ or failure of the parent to furnish hospital registration number $(n=9)$. Among the successfully contacted patients, 8 were neonates, 275 were infants, 425 were in 1-10 y age group, 342 patients were in 11-18 y age group and 29 patients were beyond $18 \mathrm{y}$ of age. Echocardiogram report was available in 1019 (94.4\%) patients. Table 2 shows the breakup of patients (seen through teleconsultation) into broad hemodynamic subgroups of CHDs. Table 3 shows the various investigations that were advised and done in the local vicinity healthcare facility to help in teleconsultation.

Cyanotic CHD: A large proportion $(24.5 \%, \mathrm{n}=265)$ of the patients were cyanotic CHD with reduced pulmonary blood flow, i.e., tetralogy of Fallot (TOF) physiology. Of these patients, worsening cyanosis was present in 24 patients. These children were asked to get oxygen saturation and hemoglobin concentration measured locally. Beta-blocker medications was optimized and good hydration ensured along with iron supplementation. Fifteen patients reported cyanotic spells, and all had significantly lower saturation, compared to the earlier value. Blood transfusion was advised at local pediatric hospital in 3 children, leading to improvement in symptoms. Twelve children were called to the hospital for further management. They were admitted and underwent surgical intervention. Cyanotic CHD with increased pulmonary blood flow constituted 57 patients; 9 of them were called to hospital for evaluation and 5 of them were admitted and underwent surgical management.
Acyanotic CHD: Acyanotic CHD with increased pulmonary blood flow constituted $23.2 \%(n=251)$ cases. Advise for maintaining dental hygiene was provided to all the patients. Medications to control heart failure were optimized in $29 \mathrm{pa}-$ tients, and 5 patients with persistent heart failure symptoms were called to the hospital for evaluation; of these 1 required admission.

Valvular lesions: Among the valvular lesions (aortic stenosis, aortic regurgitation, mitral stenosis, mitral regurgitation, pulmonary stenosis), none of the patients had worsening of symptoms.

Postoperated CHD: A total of 290 (26.8\%) patients who were advised by teleconsultation had undergone cardiac surgery in the past. Eighty-nine of these had stage-I palliation, either Blalock-Taussig shunt, bidirectional Glenn, or a pulmonary artery band. Out of 290 postoperated patients, 169 (58.2\%) had no symptoms or hemodynamically significant cardiac lesion. However, 14 postoperated patients had significant hemodynamic lesions but denied any worsening of clinical symptoms.

Miscellaneous conditions: Thirty-one patients were followedup cases of dilated cardiomyopathy with severe ventricular dysfunction and one-third of them required optimization of medications to control heart failure. Eleven patients had idiopathic pulmonary artery hypertension and 2 of these required medication optimization. Six patients had Eisenmenger syndrome and none had any worsening symptoms. Fourteen patients had arrhythmias well controlled on medical therapy. Two patients with univentricular palliation (post-Fontan surgery) had hemoptysis. Out of these, 1 was admitted, and the other was locally managed with telephonic advice.
Table 2 Breakup of patients (consulted through telecommunication) into broad hemodynamic subgroups

\begin{tabular}{lc}
\hline Hemodynamic subset & $\mathrm{N}(\%)$ \\
\hline CCHD, reduced PBF & $265(24.5 \%)$ \\
CCHD, increased PBF & $57(5.2 \%)$ \\
ACHD, increased PBF & $251(23.2 \%)$ \\
Valvar lesions & $83(7.6 \%)$ \\
Complex CCHD, asymptomatic & $13(1.2 \%)$ \\
Postoperated, asymptomatic & $188(17.4 \%)$ \\
Postoperated, symptomatic & $13(1.2 \%)$ \\
Stage-I palliation (BTS/BDG/PAB) & $89(8.2 \%)$ \\
Postcatheter intervention & $36(3.3 \%)$ \\
DCMP & $31(2.8 \%)$ \\
IPAH/Eisenmenger syndrome & $17(1.5 \%)$ \\
Arrhythmia/CHB, stable & $14(1.3 \%)$ \\
Misc (NSAA, allied specialities, HCM, postchemotherapy, hemoptysis) & $22(2 \%)$ \\
\hline
\end{tabular}

$A C H D$ Acyanotic congenital heart disease; $B D G$ Bidirectional Glenn; BTS Blalock Taussig shunt; $C C H D$ Cyanotic congenital heart disease; $C H B$ Congenital heart block; DCMP Dilated cardiomyopathy; HCM Hypertrophic cardiomyopathy; IPAH Idiopathic pulmonary artery hypertension; NSAA Non-specific aorto-arteritis; $P A B$ Pulmonary artery band; $P B F$ Pulmonary blood flow 
Table 3 Investigations and evaluations done locally to help in teleconsultation for CHD care

\begin{tabular}{ll} 
Investigations & Utility \\
\hline $\begin{array}{l}\text { Weight } \\
\begin{array}{l}\text { Oxygen } \\
\text { saturation }\end{array}\end{array}$ & $\begin{array}{c}\text { Optimization of medications according to weight } \\
\text { intervention in TOF physiology }\end{array}$ \\
Blood pressure & To assess optimal hypertension control following relief of aortic obstruction in children with coarctation of aorta and aortoarteritis \\
Hemoglobin & Decide blood transfusion in patients with heart failure and cyanotic spells \\
ASLO, CRP, & To assess recurrence of acute rheumatic fever in children with RHD \\
ESR & To assess recurrence of disease activity in patients with Takayasu arteritis \\
CRP, ESR & Optimisation of anticoagulation in patients with prosthetic heart valve implantation \\
ECG & For rhythm assessment in patients with pacemaker implantation \\
\hline
\end{tabular}

ASLO Antistreptolysin O; CHD Congenital heart disease; CRP C-reactive protein; ECG Electrocardiogram; ESR Erythrocyte sedimentation rate; PT/INR Prothrombin time/ International normalised ratio; RHD Rheumatic heart disease; TOF Tetralogy of Fallot

Overall, among all the patients who attended teleservices, medications were changed for 131 patients, and 26 patients were called to hospital. Majority of patients required no active intervention. Table 4 shows the outcomes of patients attending teleconsultation services. No active intervention was done in $75 \%$ patients. Medication change was done in $12 \%$ patients. Noncardiac issues were addressed in $4 \%$ patients. Local management of noncardiac illnesses was advised in $4 \%$ patients. Re-dating of elective catheter interventions was done in $2.5 \%$ patients. Overall, $2.5 \%$ patients were asked to visit the hospital for assessment or admission.

A total of 72 children needed admission during the 4 mo period of April to July 2020. Among these, 7 children were tested positive and were isolated and treated in designated COVID area of hospital. Among the COVID-negative children, 5 asymptomatic parents tested positive. The utilization of inpatient services also dwindled during this period. As compared to 189 patients admitted in 2019, only 66 got admitted to pediatric cardiology unit in the same period of April 1 to July 31, 2020.

As compared to 87 catheterization laboratory procedures done in 2019 , only 14 procedures were carried out in the same period in 2020. Majority of the interventions included

Table 4 Action for patients attending teleconsultations during the lockdown period

\begin{tabular}{lc}
\hline Outcome & $\mathrm{N}(\%)$ \\
\hline No active intervention & $806(74.6 \%)$ \\
Medication change & $131(12.1 \%)$ \\
Noncardiac issues addressed & $50(3.9 \%)$ \\
Local management & $40(3.7 \%)$ \\
Cath/intervention redating & $25(2.3 \%)$ \\
Patients asked to visit hospital & $26(2.4 \%)$ \\
Suture-related issues solved & $8(0.7 \%)$ \\
\hline
\end{tabular}

emergency procedures, such as balloon atrial septostomy $(n=12)$ for babies with transposition of great arteries.

During the 4-mo study period in 2019, 409 patients underwent surgery for CHD, of which 58 were emergency surgeries. However, since elective cardiac surgeries had been discontinued during this period, only 50 patients underwent surgery in corresponding 4 mo in 2020, and majority of surgeries $(n=35)$ performed were emergency surgeries. Surgery for transposition of great arteries (arterial switch operation) $(n=20)$, repair of total anomalous pulmonary venous return $(\mathrm{n}=9)$, and aorto-pulmonary shunts for TOF physiology $(n=6)$ constituted majority of surgeries that were performed during COVID pandemic.

\section{Discussion}

COVID-19 pandemic has left a huge impact on the practice of pediatric cardiology. From a dedicated pediatric cardiology unit in North India, the authors share their experience of patient management in the 4 mo following the initiation of national lockdown. During the initial 4 mo of pandemic, there was a huge reduction in inpatient admissions, as well as catheter and surgical interventions. Studies have reported up to $61 \%$ fall in cardiac surgeries during COVID-19 as compared to similar time during previous year [8]. Korun et al., from Turkey, report congenital cardiac surgery reduction during the pandemic to $2 / 3$ of that during the preceding year [9].

In the study unit, the number of patients availing successful teleconsultation was 1079 , which was $15 \%$ of the patients attending physical outpatient consultations during similar period last year. Although this number is very less as compared to the physical OPD attendance during similar period in 2019, telecommunication services proved to be a boon and helped to a great extent in patient management during COVID-19 
pandemic when actual physical patient contact was not possible due to logistic issues. According to Telecom Regulatory Authority of India (TRAI), the number of wireless telephone subscribers in India was approximately 85\% in 2018 [10]. This factor has helped in telemedicine during the current pandemic. Practice guidelines for teleconsultation were proposed by Ministry of Health and Family Welfare (MOHFW), Government of India for local use [11]. Many other subspecialties have also resorted to the use of teleservices for outpatient care [12-14]. By following teleconsultation, many children could be taken care of, which would have suffered otherwise. Furthermore, local evaluations and investigations helped save unnecessary travel to tertiary institute.

Changed workflow patterns have to be proposed to prevent disease transmission among patients and healthcare workers [2]. For inpatient care, methods of triaging have been proposed [15]. All over the world, such changes in functioning of inpatient pediatric units might be needed so that the transmission chain of COVID-19 does not propagate and hospital wards/ICUs do not become COVID-19 hotspots. As the number of CHD surgeries and interventions have reduced, prioritization for interventions has been proposed [3]. It cannot be overemphasized that preventive measures are must for the CHD population, and precautions to be taken by the patients have been recommended [16]. Re-introduction of CHD care services has brought a new normal approach by the utilisation of teleconsultation services. But many measures are required for managing the delays caused due to the interim lag phase [17].

Such teleconsultation model can be continued in future also as travel restrictions and social distancing may continue for a significant duration of time. Storing and updating the details of patient's investigations in hospital systems electronically has been of great value. It is heartening to note that such an organized teleconsultation facility may be run by a public institution even in a low-middle-income country. The use of mobile applications to share patient records and videos for assessing the patient-reported symptoms has also helped, given the wide availability of smartphones in the population. The authors believe that telecommunication is going to play a role of key importance in the current time and the future to come. Nonessential physical contact should continue to remain minimal.

Patients with CHD need continued and lifelong care. The patients visiting outpatient department can be broadly categorized into three subgroups. The first subgroup includes patients on medical follow-up. They need evaluation for progression of the disease severity (e.g., progression of stenotic or regurgitant valvular lesion) and monitoring of complications (e.g., occurrence of aortic regurgitation in a ventricular septal defect, etc.). The second subgroup are patients awaiting intervention till the appropriate timing is reached, with close watch on symptoms in the interim period which may indicate earlier need of intervention (e.g., atrial septal defect in an infant, TOF in a neonate). The third subgroup includes postintervention patients, who are followed up for rhythm evaluation, prosthetic valve, and ventricular function assessment, and monitoring of long-term events (e.g., conduit stenosis, pulmonary regurgitation, recurrence of coarctation of aorta, etc.). Many of these patients may not need to visit physical OPD at tertiary care facility to get the investigations viz. chest radiograph, ECG or oxygen saturation which are often available locally. However, some patients need physical examination and specialized investigations which are possible only by contact visit. In the postpandemic era, the timing of physical visit can be adjusted after knowing the symptoms by teleconsultation and investigations can be dated telephonically, thus reducing the number of physical visits required.

\section{Conclusion}

The utilization of cardiac services for CHD has reduced significantly during COVID-19 pandemic, both for out- and inpatients. Teleconsultation services have streamlined the follow-up care to some extent and have helped in noncontact triaging of these patients for further care.

Authors' Contribution AS conceived the idea of study. All authors contributed to the study design. Material preparation, data collection and analysis were performed by SSa and SSh. The first draft of the manuscript was written by SSa and AS, SKG, SR, SSh, SSK provided important intellectual content on the manuscript. All authors read and approved the final manuscript. All authors agree to be accountable for all aspects of the work in ensuring that questions related to the accuracy or integrity of any part of the work are appropriately investigated and resolved. AS will act as guarantor for this paper.

\section{Declarations}

Ethics Approval This study was performed in line with the principles of the Declaration of Helsinki. Approval was granted by the Institute Ethics Committee for Postgraduate Research, All India Institute of Medical Sciences, Ansari Nagar, 110029, New Delhi, India (Date: 21-10-2020/ Ref No.: IECPG-588/21.10.2020.)

Consent to Participate and Consent to Publish In view of retrospective data collection, consent waiver was obtained from the Ethics committee. Personal data of none of the study participants is shared. Participant identity is not disclosed.

Conflict of Interest None.

\section{References}

1. Iyer KS. Impact of the COVID-19 pandemic on pediatric cardiac care in India: time for action. Ann Pediatr Cardiol. 2020;13:183-5.

2. Dilli D, Taşoğlu I. Perioperative care of the newborns with CHDs in the time of COVID-19. Cardiol Young. 2020;30:946-54. 
3. Stephens EH, Dearani JA, Guleserian KJ, et al. COVID-19: crisis management in congenital heart surgery. World J Pediatr Congenit Heart Surg. 2020;11:395-400.

4. Farooqi KM, Ghoshhajrab BB, Shaha AM, et al. Recommendations for risk stratified use of cardiac computed tomography for congenital heart disease during the COVID-19 pandemic. J Cardiovasc Comput Tomogr. 2020;14:291-3.

5. Hemphill NM, Kuan MTY, Harris KC. Reduced physical activity during COVID-19 pandemic in children with congenital heart disease. Can J Cardiol. 2020;36:1130-4.

6. De Rosa S, Spaccarotella C, Basso C, et al. Reduction of hospitalizations for myocardial infarction in Italy in the COVID-19 era. Eur Heart J. 2020;41:2083-8.

7. Mafham MM, Spata E, Goldacre R, et al. COVID-19 pandemic and admission rates for and management of acute coronary syndromes in England. Lancet. 2020;396:381-9.

8. Casey L, Khan N, Healy DG. The impact of the COVID-19 pandemic on cardiac surgery and transplant services in Ireland's National Centre. Ir J Med Sci. 2020;190:13-7. https://doi.org/10. 1007/s11845-020-02292-6.

9. Korun O, Yurdakök O, Arslan A, et al. The impact of COVID-19 pandemic on congenital heart surgery practice: an alarming change in demographics. J Card Surg. 2020;35:2908-12.

10. Telecom Regulatory Authority of India. Government of India. Wireless Data Services in India, an analytical report. Available at: https://trai.gov.in/sites/default/files/Wireless_Data_Service Report21082019_0.pdf. Accessed 25 October 2020.

11. Ministry of Health and Family Welfare. Telemedicine Practice Guidelines. Enabling Registered Medical Practitioners to provide healthcare using telemedicine. Available at: https://www.mohfw. gov.in/pdf/Telemedicine.pdf. Accessed 25 October 2020.

12. Deshmukh AV, Badakere A, Sheth J, Bhate M, Kulkarni S, Kekunnaya R. Pivoting to teleconsultation for paediatric ophthalmology and strabismus: our experience during COVID-19 times. Indian J Ophthalmol. 2020;68:1387-91.

13. Ganguly S, Bakhshi S. Teleconsultations and shared care in pediatric oncology during COVID-19. Indian J Pediatr. 2020;88:1-2. https://doi.org/10.1007/s12098-020-03499-2. Accessed 25 October 2020.

14. Sundriyal D, Sehrawat A, Kumar P, Bhandari R. Impact of COVID-19 pandemic on oncology practices during nationwide lockdown period: a single centre experience and the way forward. J Assoc Physicians India. 2020;68:48-50.

15. Lastinger LT, Daniels CJ, Lee M, Sabanayagam A, Bradley EA. Triage and management of the ACHD patient with COVID-19: a single center approach. Int J Cardiol. 2020;320:178-82.

16. Radke RM, Frenzel T, Baumgartner H, Diller GP. Adult congenital heart disease and the COVID-19 pandemic. Heart. 2020;106:13029.

17. Dearani JA, Stephens EH, Guleserian KJ, et al. COVID-19: FAQs - congenital heart surgery recovery and defining a "new normal". World J Pediatr Congenit Heart Surg. 2020;11:548-56.

Publisher's Note Springer Nature remains neutral with regard to jurisdictional claims in published maps and institutional affiliations. 